\title{
Detailed plasma potential measurements in a radio-frequency expanding plasma obtained from various electrostatic probes
}

\author{
T. Lafleur,, ${ }^{a)}$ C. Charles, and R. W. Boswell \\ Space Plasma, Power, and Propulsion Group, Research School of Physics and Engineering, \\ The Australian National University, Canberra ACT 0200, Australia
}

(Received 28 February 2009; accepted 7 April 2009; published online 29 April 2009)

\begin{abstract}
On-axis plasma potential measurements have been made with an emissive probe in a low pressure $(0.044 \mathrm{~Pa}) \mathrm{rf}$ expanding plasma containing an ion beam. The beam is detected with a retarding field energy analyzer (RFEA), and is seen to disappear at high pressure $(0.39 \mathrm{~Pa})$. The emissive probe measurements are in very good agreement with corresponding measurements made with two separate RFEAs, and the results indicate that the floating potential of the strongly emitting probe gives an accurate measure of the plasma potential under the present conditions. (C) 2009 American Institute of Physics. [DOI: 10.1063/1.3125314]
\end{abstract}

Recent studies with electric double layers, ${ }^{1-3}$ and ion beam formation, ${ }^{4-6}$ have reignited the interest in low pressure expanding plasmas, in connection with astrophysical phenomena in the field of space science, ${ }^{7}$ and particularly as a means of producing thrust in propulsion applications. ${ }^{8}$ One consequence of this research has been the development of a new type of plasma thruster, the helicon double layer thruster (HDLT) ${ }^{8}$ In order to fully characterize such thrusters, however, detailed spatial mappings of the system are becoming necessary ${ }^{9}$ to help clarify operational modes and underlying physical phenomena, as have been performed in Hall-effect thrusters. ${ }^{10}$ Until now, retarding field energy analyzers (RFEAs) have been used almost exclusively to determine the plasma potential in the HDLT, but due to its design, it can at times be bulky, perturbative, or restrictive. By contrast, emissive probes (EPs) can be made much smaller, and are seen as the best diagnostic tools for finding the plasma potential. ${ }^{11,12}$ EPs are increasingly being used in a range of fields, including fusion experiments, where they offer a number of advantages over cold probes (such as Langmuir probes). ${ }^{13,14}$ In this brief communication we take EP measurements of the potential in a low pressure rf expanding plasma containing an ion beam, and perform a direct comparison with corresponding RFEA results.

Measurements are made in the Piglet helicon reactor shown in Fig. 1. It consists of an $18 \mathrm{~cm}$ long, $13.6 \mathrm{~cm}$ inner diameter Pyrex tube, attached contiguously to a $28.8 \mathrm{~cm}$ long, $30 \mathrm{~cm}$ diameter aluminum diffusion chamber. The Pyrex tube is open at both ends, with the back end being terminated by a grounded metal grid, to which a turbomolecular/rotary pump system are connected. A rf power supply and matching network provide a constant power of $200 \mathrm{~W}$ at $13.56 \mathrm{MHz}$ to a double saddle field antenna (extending from $z=-16 \mathrm{~cm}$ to $z=-5 \mathrm{~cm}$ ) surrounding the Pyrex tube. Two solenoids, of $\sim 500$ turns each, provide a diverging magnetic field, with a maximum strength of $\sim 0.016 \mathrm{~T}$ in the source $(z=-14 \mathrm{~cm})$ and a few millitorr in the diffusion chamber. For these conditions the coil closest to

\footnotetext{
${ }^{a)}$ Electronic mail: trevor.lafleur@anu.edu.au
}

the pump (source coil) is supplied with 4 A while the second coil (exhaust coil) is supplied with 3 A. Argon gas is introduced into the system via an inlet in the side of the diffusion chamber, and the pressure is measured using an ion gauge and a baratron gauge attached to the back plate of the chamber.

Plasma properties in Piglet are measured with a number of electrostatic probes inserted into the reactor via ports in the back plate of the diffusion chamber. The plasma potential is measured with an emissive probe consisting of a 0.125 $\mathrm{mm}$ diameter tungsten wire inserted into two holes of a 5 $\mathrm{mm}$ diameter four bore ceramic tube. Copper wires placed within the ceramic holes provide the heating current which is supplied from a dc power supply connected across an isolation transformer (ensuring that the heating current does not form part of the measured current). Additional thicker tungsten wires are tightly packed between the filament and copper wires, to help provide strong electrical contact, and cooling. The chosen filament diameter is smaller than the smallest electron gyroradius within Piglet, so as to reduce space charge effects. ${ }^{10,11}$ The probe tip is aligned such that the plane of the loop is perpendicular to Piglet's axis. This

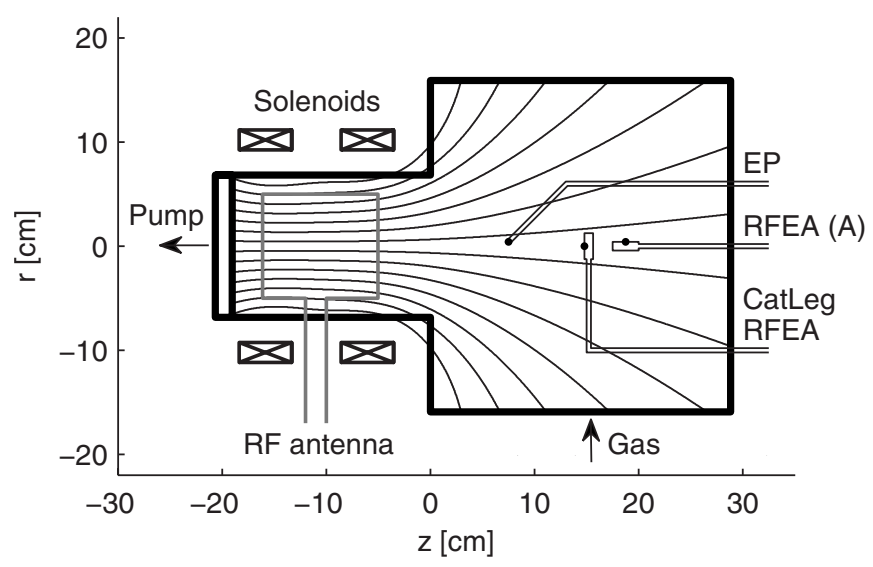

FIG. 1. Schematic of the Piglet reactor showing the plasma source tube, axial solenoids with corresponding magnetic field lines, and diffusion chamber with diagnostics inserted through the backplate. 
(a)
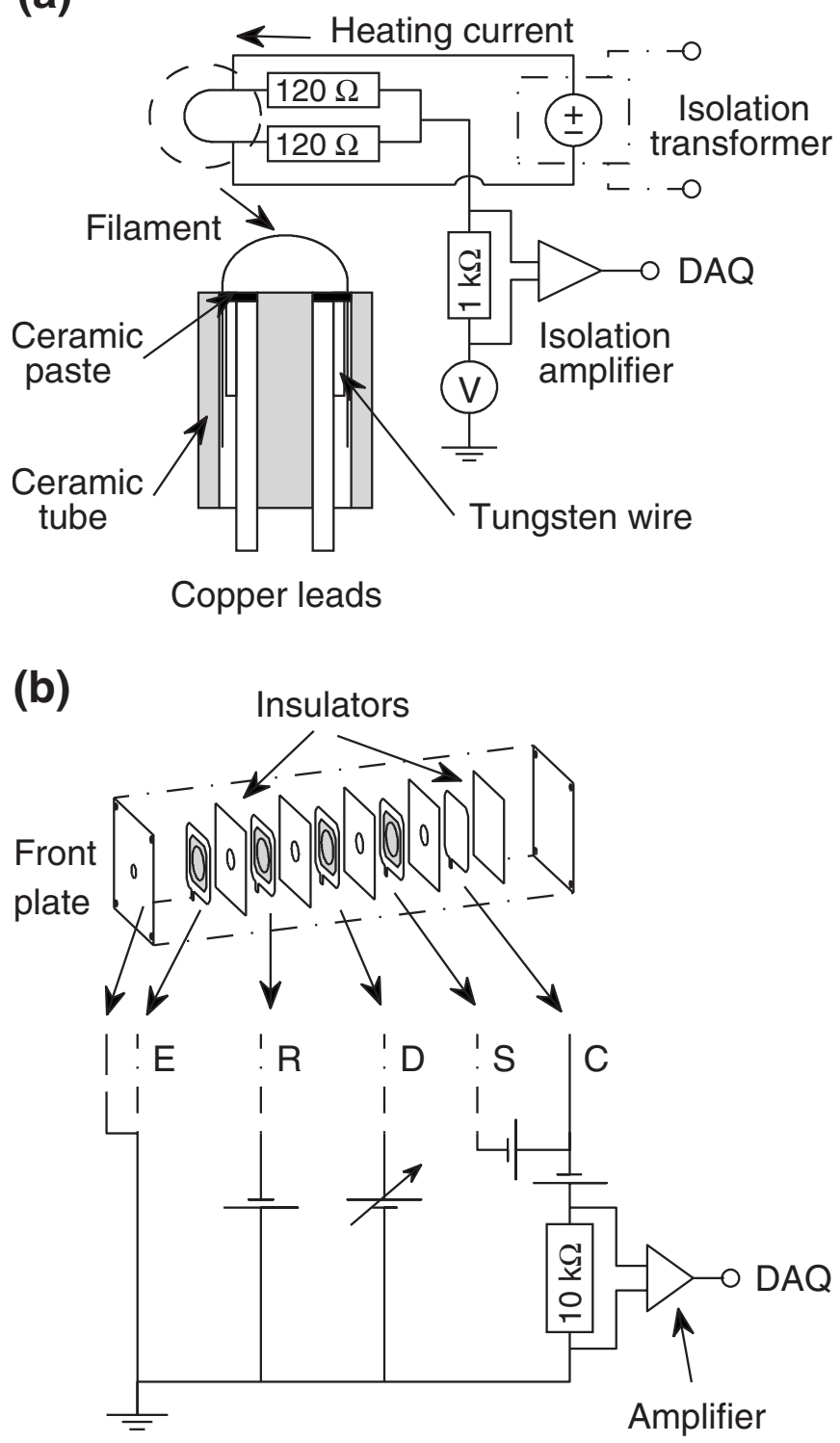

FIG. 2. (a) Schematic of the emissive probe showing probe tip design, and sweeping circuit. The ceramic tube is attached to a hollow grounded metal shaft via a grub screw. (b) Schematic of the RFEA head and associated circuitry, showing earth grid (E: $0 \mathrm{~V})$, repellor $(\mathrm{R}:-90 \mathrm{~V})$, discriminator (D: $0-80 \mathrm{~V})$, secondary $(\mathrm{S}:-16 \mathrm{~V})$, and collector plate $(\mathrm{C}:-9 \mathrm{~V})$. The plasma enters the RFEA from the orifice in the grounded front plate.

helps to reduce uncertainty in measured axial potentials, by ensuring that the entire filament sees the same potential (since the system is approximately cylindrically symmetric), as opposed to if the loop were parallel to the axis, where now the ends of the filament effectively see slightly different potentials due to the axial plasma potential gradient present within the reactor. Figure 2(a) shows a schematic of the probe tip, together with the sweeping circuit used (see below). A retarding field energy analyzer [RFEA (A) in Fig. 1] with aperture facing the walls of the reactor, is used to take measurements of the local plasma potential along the $z$ axis. ${ }^{15}$ A second RFEA (CatLeg RFEA in Fig. 1) with aperture facing the upstream source tube and with a support shaft consisting of a $90^{\circ}$ bend, is used to detect the presence of an ion beam, as well as to take additional downstream measure-

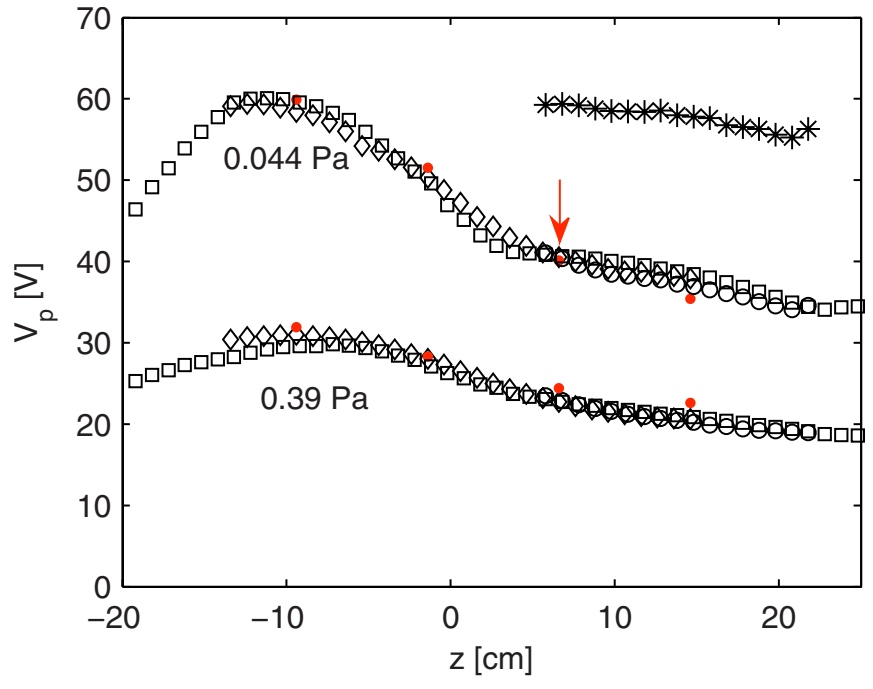

FIG. 3. (Color online) Axial plasma potentials for the two pressure cases measured with the strongly emitting floating EP (open diamonds), EP using the inflection point method (closed red circles), RFEA (a) (open squares), CatLeg RFEA (open circles), and the beam potentials measured with the CatLeg RFEA (stars). The gray arrow (red online) at $z=6.8 \mathrm{~cm}$ corresponds to the probe positions of Figs. 4(a)-4(d).

ments of the potential. ${ }^{15}$ The RFEA probe head consists of four nickel grids spot welded onto copper supports, a grounded front plate, and a collector plate. Thin insulators are placed between adjacent copper supports, and the collector plate. By applying certain voltages [see Fig. 2(b)] to each of the grids, plasma electrons can be repelled, and the energy of the ions entering the front plate can be discriminated. Both RFEAs are similar in design too those in Refs. 16 and 17 (where further construction details are given) but with slightly different voltages applied to the grids, and no water cooling present. A schematic of the RFEA, together with the related circuitry is shown in Fig. 2(b).

To demonstrate the pressure dependence of ion beam formation in the present reactor, two conditions are chosen, a high pressure $0.39 \mathrm{~Pa}(2.9 \mathrm{mTorr})$ case, and a low pressure $0.044 \mathrm{~Pa}$ ( $0.33 \mathrm{mTorr})$ case. The emissive probe is operated using two methods. In the first method, the floating potential of the strongly emitting probe is measured with a high impedance multimeter. ${ }^{14,18}$ The correct heating current is determined by observing the floating potential as a function of the heating current. After initial emission, the floating potential eventually saturates with increasing heating current. This floating potential is then taken as the plasma potential. Heating currents between 2.6 and $3 \mathrm{~A}$ were needed for saturation to occur, which resulted in a voltage drop of $\sim 2.5 \mathrm{~V}$ across the filament, so that measurements are expected to have an uncertainty of $\pm 1.25 \mathrm{~V}$. In the second method, the inflection point of the differentiated current-voltage $I(V)$ curve is tracked as the electron emission is varied. The plasma potential is then inferred by extrapolation of the inflection point to zero emission using the method described in Refs. 19 and 20. The current is measured across a $1 \mathrm{k} \Omega$ sense resistor with an isolation amplifier and data acquisition system, while the bias voltage is supplied with a standard sweeping circuit, as shown in Fig. 2(a). 
The RFEAs are used to measure the on-axis collector current versus discriminator voltage characteristic $I_{c}\left(V_{d}\right)$ and from its derivative the ion energy distribution function (IEDF) can be established. Gaussian functions are then fitted to the IEDF using a previously described deconvolution process. ${ }^{15}$ Since the aperture of RFEA (A) faces the reactor walls, it can only see the local ion population, and thus the IEDF is fitted with a single Gaussian centered on the local plasma potential. ${ }^{15}$ In the presence of an ion beam, the CatLeg RFEA can see a second population of higher energy ions, and a second peak is observed in the IEDF.

Figure 3 shows the axial potential profiles measured with both the EP and RFEAs for the two pressures cases, while Fig. 4 shows typical probe characteristics at $z=6.8 \mathrm{~cm}$ (red arrow in Fig. 3) for $0.044 \mathrm{~Pa}$. For both pressure cases, deviation between the measured probe potentials is lower in some regions than others, but in general, very good agreement is seen throughout. An ion beam is only observed at $0.044 \mathrm{~Pa}$, once again showing the dependence of ion beam formation with pressure, consistent with previous observations in similar reactors and conditions. ${ }^{1,5,6,21}$ The observed ion beam energy of $\sim 60 \mathrm{~V}$ is consistent with the peak upstream potential in the middle of the source measured with both RFEA (A), and the EP. Care needs to be taken with the EP strongly emitting in the low pressure case, especially in the downstream region, as the filament is susceptible to damage or failure (possibly due to the presence of the ion beam). Once damaged, the resistance of the filament is observed to increase, and it no longer necessarily emits within the plasma, but can now emit within the ceramic tube. If the connections within the probe tip are not sufficiently reliable, thermal expansion during operation can cause unstable contact along the length of the filament. ${ }^{22}$ To prevent this, a very tight connection must be made between the filament and copper/tungsten wires. Figure 4(a) shows the IEDF at $z$ $=6.8 \mathrm{~cm}$ from RFEA (A), clearly showing a single ion population at the local plasma potential, while the existence of the ion beam can be seen in the CatLeg RFEA IEDF of Fig. 4(b), where the beam energy is $\sim 18 \mathrm{~V}$ above the local ion population.

The inflection point method of EPs is seen as the more reliable method for measuring the plasma potential. ${ }^{11,12} \mathrm{Un}$ fortunately, this method is slow and time consuming, and does not lend itself well to large data sets or detailed twodimensional spatial mappings. Nevertheless, as this is one of the first measurements of the plasma potential with an EP in an rf expanding plasma, it is important to confirm the EP operation with the inflection point method, to establish confidence in future results. This is particularly true since space charge effects may cause error in the measured plasma potential depending on the operating conditions. In some cases a virtual cathode can form within the probe sheath due to space charge effects associated with strong emission. ${ }^{13,23}$ This can then cause the floating potential to be lower than the actual plasma potential. There is also evidence that space charge effects can produce a variation in the electron saturation current, indicating that strong emission might in some cases perturb the plasma itself. ${ }^{24,25}$ However, as seen for the two pressure cases in Fig. 3, both EP methods show good
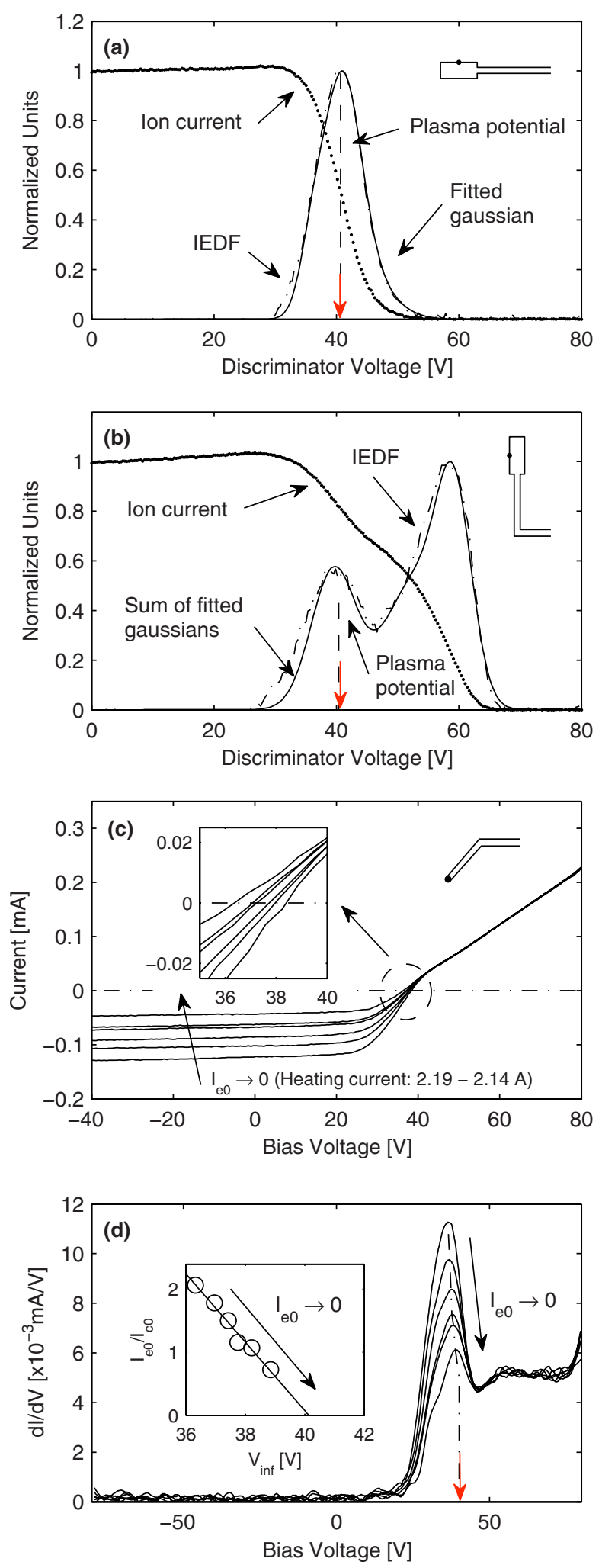

FIG. 4. (Color online) Probe characteristics at $z=6.8 \mathrm{~cm}$ : (a) Normalized ion current and IEDF for RFEA (A). (b) Normalized ion current and IEDF for the CatLeg RFEA. (c) Swept EP $I(V)$ characteristics at a number of emission levels. The inset figure shows a magnified image of the where the probe characteristics cross the zero current line. Heating currents for the characteristics shown range from 2.19 to $2.14 \mathrm{~A}$, in steps of $0.01 \mathrm{~A}$. (d) Corresponding derivatives of the $I(V)$ characteristics in (c) showing peaks at the inflection points. The inset figure shows the extrapolation of the inflection point potential $\left(V_{\text {inf }}\right)$ to zero emission $\left(I_{e 0} \rightarrow 0\right)$. The gray arrows (red online) in (a)-(d) above represent the floating potential of the strongly emitting EP at the corresponding position. 


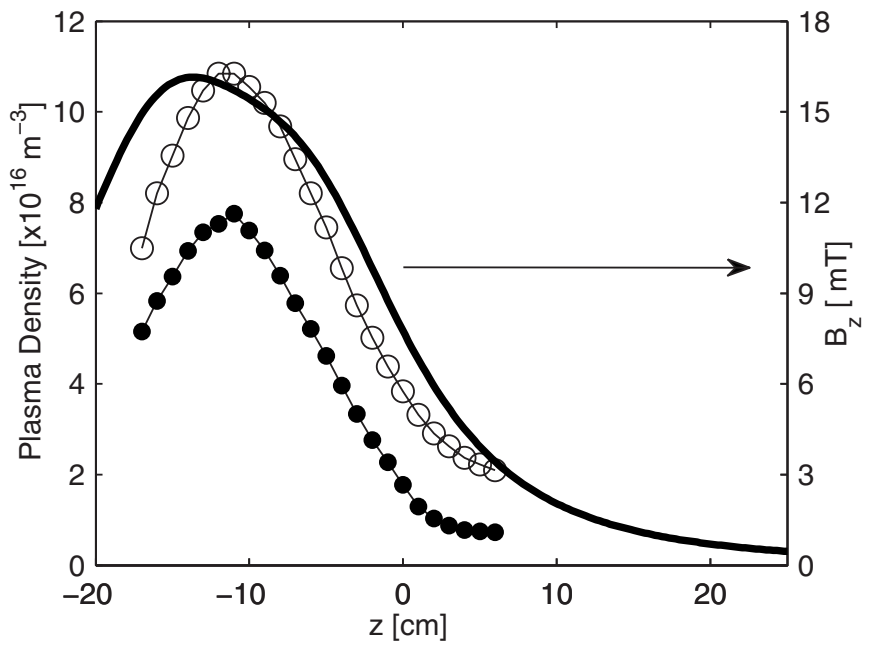

FIG. 5. On-axis plasma density profiles obtained with a Langmuir probe for $0.044 \mathrm{~Pa}$ (filled circles) and $0.39 \mathrm{~Pa}$ (open circles); calculated axial magnetic field strength $\left(B_{z}\right)$ with $4 \mathrm{~A}$ in the source coil, and $3 \mathrm{~A}$ in the exhaust coil.

agreement (closed red circles, and corresponding open diamonds), giving us confidence that the floating potential of the strongly emitting EP is a reasonable measurement of the plasma potential. Figure 4(c) shows representative $I(V)$ curves for the EP as the heating current is varied, while Fig. 4(d) shows the corresponding derivative of each curve, where a broad single peak is clearly seen. The plasma potential is found by plotting the inflection point potentials $\left(V_{\text {inf }}\right)$ as a function of the probe emission $\left(I_{e 0}\right)$ to collection current $\left(I_{c 0}\right)$ ratio, and extrapolating to zero emission. An example of this is seen as the inset graph in Fig. 4(d). At $z=14.6 \mathrm{~cm}$, as the emission current is reduced, the single peak splits into two peaks, as is normally characteristic of rf driven systems, and becomes better resolved as the emission current is further reduced. At this position the peak-to-peak amplitude is $\sim 7 \mathrm{~V}$, while at $z=-9.2 \mathrm{~cm}$ it is $\sim 5 \mathrm{~V}$. The plasma potentials from the probe characteristics in Fig. 4 match well with the floating potential (red arrows) of the strongly emitting EP at the corresponding position.

Figure 5 shows the axial plasma density profiles for both pressure cases, together with the magnetic field strength variation. The densities are obtained with a Langmuir probe in ion saturation mode, using a downstream electron temperature of $2.5 \mathrm{eV}$ at $0.39 \mathrm{~Pa}$, and $5 \mathrm{eV}$ at $0.044 \mathrm{~Pa}$, measured with a rf compensated Langmuir probe ${ }^{26}$ (both probes are inserted into the same port as for the EP, but are not shown in Fig. 1 for clarity). The densities are found to peak close to the center of the antenna $(z=-10.5 \mathrm{~cm})$. It is interesting to note that the potential does not drop as sharply as seen in previous investigations with similar reactors. ${ }^{1,21}$
Aside from the obvious geometric differences, the magnetic field does not display two separate peaks, since the coils are located fairly close to each other. Additionally, the back wall of the source tube is grounded, not insulating, so it cannot float. Previous work suggests these factors are significant, and they will be investigated in further work.

In summary, an emissive probe has been used to measure the axial plasma potential profile of a rf expanding plasma, using two separate methods, both in the presence and absence of an ion beam. The results are in very good agreement with corresponding measurements taken with two RFEAs, and show that the plasma potential can be accurately measured with a strongly emitting floating probe.

${ }^{1}$ C. Charles and R. W. Boswell, Appl. Phys. Lett. 82, 1356 (2003).

${ }^{2}$ S. A. Cohen, N. S. Siefert, S. Strange, R. F. Boivin, E. E. Scime, and F. M. Levinton, Phys. Plasmas 10, 6 (2003).

${ }^{3}$ X. Sun, A. M. Keesee, C. Biloiu, E. E. Scime, A. Meige, C. Charles, and R. W. Boswell, Phys. Rev. Lett. 95, 025004 (2005).

${ }^{4}$ A. Fruchtman, Phys. Rev. Lett. 96, 065002 (2006).

${ }^{5}$ C. S. Corr, J. Zanger, R. W. Boswell, and C. Charles, Appl. Phys. Lett. 91, 241501 (2007).

${ }^{6}$ K. Takahashi, K. Oguni, H. Yamada, and T. Fujiwara, Phys. Plasmas 15, 084501 (2008)

${ }^{7}$ R. W. Boswell, E. Marsch, and C. Charles, Astrophys. J. 640, L199 (2006).

${ }^{8}$ C. Charles, Plasma Sources Sci. Technol. 16, R1 (2007).

${ }^{9}$ W. Cox, C. Charles, R. W. Boswell, and R. Hawkins, Appl. Phys. Lett. 93, 071505 (2008).

${ }^{10}$ J. Haas and A. D. Gallimore, Phys. Plasmas 8, 652 (2001).

${ }^{11}$ N. Hershkowitz and M. H. Cho, J. Vac. Sci. Technol. A 6, 2054 (1988).

${ }^{12}$ N. Hershkowitz, in Plasma Diagnostics, edited by O. Auciello and D. L. Flamm (Academic, San Diego, 1989), p. 146.

${ }^{13}$ R. Schrittwieser, J. Adámek, P. Balan, M. Hron, C. Ioniță, K. Jakubka, L. Kryška, E. Martines, J. Stöckel, M. Tichý, and G. Van Oost, Plasma Phys. Controlled Fusion 44, 567 (2002).

${ }^{14}$ R. Schrittwieser, C. Ioniță, P. C. Balan, J. A. Cabral, F. H. Figueiredo, V. Pohoața, and C. Varandas, Contrib. Plasma Phys. 41, 494 (2001).

${ }^{15}$ C. Charles and R. W. Boswell, Phys. Plasmas 11, 1706 (2004).

${ }^{16}$ D. L. Rudakov, M. G. Shats, R. W. Boswell, C. Charles, and J. Howard, Rev. Sci. Instrum. 70, 476 (1999).

${ }^{17}$ G. D. Conway, A. J. Perry, and R. W. Boswell, Plasma Sources Sci. Technol. 7, 337 (1998).

${ }^{18}$ R. E. Kemp and J. M. Sellen, Jr., Rev. Sci. Instrum. 37, 455 (1966).

${ }^{19}$ J. R. Smith, N. Hershkowitz, and P. Coakley, Rev. Sci. Instrum. 50, 210 (1979).

${ }^{20}$ E. Y. Wang, N. Hershkowitz, T. Intrator, and C. Forest, Rev. Sci. Instrum. 57, 2425 (1986).

${ }^{21}$ O. Sutherland, C. Charles, N. Plihon, and R. W. Boswell, Phys. Rev. Lett. 95, 205002 (2005).

${ }^{22}$ A. Siebenförcher and R. Schrittwieser, Rev. Sci. Instrum. 67, 849 (1996).

${ }^{23}$ M. Y. Ye and S. Takamura, Phys. Plasmas 7, 3457 (2000).

${ }^{24}$ A. Marek, M. Jilek, I. Pickov, P. Kudrna, M. Tichý, R. Schrittwieser, and C. Ioniță, Contrib. Plasma Phys. 48, 491 (2008).

${ }^{25}$ A. Marek, I. Picková, P. Kudrna, M. Tichý, R. P. Apetrei, S. B. Olenici, R. Gstrein, R. Schrittwieser, and C. Ioniță, Czech. J. Phys. 56, B932 (2006).

${ }^{26}$ K. Takahashi, C. Charles, and R. W. Boswell, Phys. Plasmas 14, 114503 (2007). 